\title{
Long-term prognostic outcomes in patients with haemoptysis
}

\author{
Michele Mondoni ${ }^{1 *}$, Paolo Carlucci ${ }^{1}$, Giuseppe Cipolla ${ }^{2}$, Matteo Pagani ${ }^{3}$, Francesco Tursi ${ }^{4}$, Alessandro Fois ${ }^{5}$, \\ Pietro Pirina ${ }^{5}$, Sara Canu ${ }^{5}$, Stefano Gasparini ${ }^{6}$, Martina Bonifazi ${ }^{6}$, Silvia Marani ${ }^{7}$, Andrea Comel ${ }^{8}$, Laura Saderi ${ }^{9}$, \\ Sabrina De Pascalis ${ }^{1}$, Fausta Alfano ${ }^{1}$, Stefano Centanni ${ }^{1}$ and Giovanni Sotgiu ${ }^{9}$
}

\begin{abstract}
Background: Haemoptysis is a challenging symptom that can be associated with potentially life-threatening medical conditions. Follow-up is key in these patients to promptly detect new or misdiagnosed pathologic findings. Few prospective studies have evaluated long-term prognostic outcomes in patients with haemoptysis. Furthermore, the role played by antiplatelet and anticoagulant drugs on mortality and recurrence rates is unclear. The aim of this study was to assess mortality after 18 months of follow-up. Furthermore, the incidence of recurrence and the risk factors for recurrence and death were evaluated (including the role played by anticoagulant and antiplatelet drugs).
\end{abstract}

Methods: Observational, prospective, multicentre, Italian study.

Results: 451/606 (74.4\%) recruited patients with haemoptysis completed the 18 months follow-up. 22/604 (3.6\%) diagnoses changed from baseline to the end of the follow-up. 83/604 (13.7\%) patients died. In 52/83 (62.7\%) patients, death was the outcome of the disease which caused haemoptysis at baseline. Only the diagnosis of lung neoplasm was associated with death (OR (95\%Cl): 38.2 (4.2-347.5); p-value: 0.0001). 166 recurrences were recorded in 103/604 (17\%) patients. The diagnosis of bronchiectasis was significantly associated with the occurrence of a recurrence (OR (95\% Cl): 2.6 (1.5-4.3)); p-value < 0.0001). Anticoagulant, antiaggregant, and anticoagulant plus antiaggregant drugs were not associated with an increased risk of death and recurrence.

Conclusions: Our study showed a low mortality rate in patients with haemoptysis followed-up for 18 months. Pulmonary malignancy was the main aetiology and the main predictor of death, whereas bronchiectasis was the most frequent diagnosis associated with recurrence. Antiplatelet and/or anticoagulant therapy did not change the risk of death or recurrence. Follow-up is recommended in patients initially diagnosed with lower airways infections and idiopathic bleeding.

Trial registration: NCT02045394

Keywords: Haemoptysis, Recurrence, Lung cancer, Bronchoscopy, Anticoagulant, Antiplatelet, Mortality, Bronchiectasis

\footnotetext{
*Correspondence: michele.mondoni@asst-santipaolocarlo.it

${ }^{1}$ Respiratory Unit, ASST Santi Paolo e Carlo, San Paolo Hospital,

Department of Health Sciences, Università degli Studi di Milano, Via A. Di Rudinì n.8, 20142 Milan, Italy

Full list of author information is available at the end of the article
}

\section{Background}

Haemoptysis is a challenging symptom that can be associated with potentially life-threatening medical conditions $[1,2]$. Recent studies have shown that lung cancer, bronchiectasis, and lower respiratory tract infections are the most frequent aetiologies [2-8]. However, despite an accurate initial work-up, a subgroup of patients with original author(s) and the source, provide a link to the Creative Commons licence, and indicate if changes were made. The images or other third party material in this article are included in the article's Creative Commons licence, unless indicated otherwise in a credit line to the material. If material is not included in the article's Creative Commons licence and your intended use is not permitted by statutory regulation or exceeds the permitted use, you will need to obtain permission directly from the copyright holder. To view a copy of this licence, visit http://creativecommons.org/licenses/by/4.0/. The Creative Commons Public Domain Dedication waiver (http://creativeco mmons.org/publicdomain/zero/1.0/) applies to the data made available in this article, unless otherwise stated in a credit line to the data. 
haemoptysis does not have an aetiological diagnosis (i.e., idiopathic or cryptogenic haemoptysis) [2-9]; furthermore, diagnostic changes from the baseline assessment to recurrences have been recently described $[2,9]$. In particular, lung cancers and bronchiectasis were subsequently diagnosed in patients with an initial diagnosis of idiopathic haemoptysis and lower respiratory tract infection $[2,10]$. In this context, follow-up is key to detecting new or misdiagnosed pathologic findings (e.g., lung malignancy) $[2,6,8]$.

Several factors might influence the long-term prognostic outcomes of patients with haemoptysis. Few prospective studies have evaluated the survival rate, the mortality-related risk factors, or the incidence of recurrence and its associated variables [7, 8]. Furthermore, the role played by antiplatelet and anticoagulant drugs is still unclear.

The primary aim of this study was to assess the mortality after 18 months of follow-up; furthermore, the incidence of recurrence and the risk factors for recurrence and death were evaluated. The role of anticoagulant and antiplatelet drugs on these outcomes was also studied.

\section{Materials and methods Study design}

This is a secondary analysis of an observational, prospective, multicentre, Italian study aimed at evaluating the epidemiology of haemoptysis in Italy and the diagnostic yield of the most frequently prescribed diagnostic techniques [5]. It was approved by the ethical committees of five Italian participating hospitals and registered at ClinicalTrials.gov (identifier: NCT02045394). Written informed consent was provided by all recruited patients [5], who were followed-up for 18 months.

One month after the recruitment and the first initial assessment, a hospital clinical re-evaluation was scheduled. After three, six, nine, twelve, and eighteen months, a phone call was planned for every patient. At each follow-up visit, information on the occurrence, timing, and severity of recurrences was collected.

In case of a recurrence, a new clinical assessment was performed; data on clinical, radiological, and endoscopic examinations, as well as on symptom management were recorded.

\section{Patients and interventions}

From July 2013 to September 2015, adult (i.e., $\geq 18$ years old) patients with haemoptysis requiring an aetiological diagnosis were considered eligible for recruitment [5] and consecutively enrolled. Exclusion criteria were the following: 1) aetiology of haemoptysis already known; 2) refusal to sign the informed consent. The follow-up period lasted from December 2015 to February 2018.
The severity of haemoptysis was graded by the first attending physician. Patients were divided into three groups based on the total amount of blood expectorated in $24 \mathrm{~h}(\mathrm{~h})[5,7]$ : mild (i.e., drops of blood to 20 millilitres $(\mathrm{ml}) / 24 \mathrm{~h}$ ), moderate (i.e., $20-500 \mathrm{ml} / 24 \mathrm{~h}$ ), severe (i.e., $>500 \mathrm{ml} / 24 \mathrm{~h}$ ).

\section{Outcome measures}

The primary outcome was the survival rate of patients with haemoptysis. Furthermore, the incidence of recurrence was calculated, and the main factors associated with recurrence and mortality were determined. The effectiveness of antiplatelet and anticoagulant drugs on these outcomes was specifically investigated. Changes in the diagnosis of haemoptysis from baseline to the end of follow-up were recorded.

\section{Statistical analysis}

Qualitative and quantitative variables were collected with an ad hoc electronic form. Qualitative variables were described with absolute and relative (percentage) frequencies, whereas quantitative variables were summarised as medians (interquartile ranges, IQR) for their non-parametric distribution. Univariate and multivariate logistic regression analysis was performed to assess the relationships between clinical, demographic, and epidemiological variables and the outcomes death and recurrence. A two-tailed p-value less than 0.05 was considered statistically significant. All the statistical computations were performed with the statistical software STATA version 16 (StatsCorp, Texas, USA).

\section{Results}

An 18 month follow-up assessment was completed by 451 out of 606 (74.4\%) patients who were recruited [5].

The initial aetiological diagnoses were previously described [5]. In addition to specific aetiological therapy, bronchial artery embolisation was performed in 13/606 (2.1\%) patients, and bronchoscopy was performed with a therapeutic aim (e.g., administration of topical vasoconstriction, Fogarty balloon, endobronchial argon plasma coagulation, and laser therapy) in 99/604 (16.4\%) patients. Oral and intravenous tranexamic acid were prescribed in 119/606 (19.6\%) patients.

$70 / 606$ (11.5\%) patients were lost to follow-up, whereas $83 / 606$ (13.7\%) died during the study period. Within one month after the enrolment 15/83 (18.1\%) patients died. $21 / 83$ (25.3\%) patients died between the first and the third month of follow-up, 14/83 (16.9\%) between the third and the sixth month, 26/83 (31.3\%) between the sixth and the twelfth month, and 7/83 (8.4\%) between the twelfth and the eighteenth month. 
Death was the outcome of the disease which caused haemoptysis at baseline in 52/83 (62.7\%) patients: 42 died due to lung cancer (eight during a recurrence), seven patients for metastatic pulmonary malignancy, two patients for pneumonia, and one for a bronchiectasis exacerbation. One patient, initially diagnosed with idiopathic haemoptysis, died during a recurrence without an identifiable cause of bleeding.

In the univariate analysis, age $>70$ years (odds ratio, OR: 9.5; p-value: 0.03 ), being male $\mathrm{r}$ (OR: 2.3; p-value: 0.005 ), moderate haemoptysis (OR: 1.9; p-value: 0.01 ), smoking history ( $\geq 10$ pack/years: OR: 3.5 ; p-value: $<0.0001 ; \geq 30$ pack/years: OR 1.9; p-values: 0.02), and pulmonary malignancy (OR: 15.6; p-value: $<0.0001$ ) were associated with an increased risk of mortality. In the multivariate analysis, only the diagnosis of lung neoplasm resulted significantly associated with the above-mentioned outcome (OR: 38.2; p-value: 0.0001) (Table 1).

From baseline to the end of the follow-up, 22/606 (3.6\%) diagnoses changed (Table 2). In particular, pulmonary malignancy was described in four cases initially
Table 2 Variation in the diagnosis from the initial assessment after 18 months of follow-up

\begin{tabular}{|c|c|c|}
\hline Initial diagnosis & $\begin{array}{l}\text { Diagnosis at the end of } \\
\text { follow-up }\end{array}$ & $\begin{array}{l}\text { Patient } \\
\text { number }\end{array}$ \\
\hline Pneumonia/lung abscess & Lung cancer & 4 \\
\hline Pneumonia/lung abscess & $\begin{array}{l}\text { Pulmonary embolism (with infarc- } \\
\text { tion) }\end{array}$ & 2 \\
\hline Pneumonia/lung abscess & Bronchiectasis & 1 \\
\hline Cryptogenic haemoptysis & Lung cancer & 4 \\
\hline Cryptogenic haemoptysis & Upper airways bleeding disease & 1 \\
\hline Cryptogenic haemoptysis & $\operatorname{ILD}$ & 1 \\
\hline Cryptogenic haemoptysis & Hematemesis & 1 \\
\hline COPD exacerbation & Bronchiectasis & 2 \\
\hline COPD exacerbation & Pulmonary malignancy & 1 \\
\hline COPD exacerbation & Lung cancer & 1 \\
\hline Acute bronchitis & Bronchiectasis & 1 \\
\hline Acute bronchitis & COPD exacerbation & 1 \\
\hline Acute bronchitis & Upper airways bleeding disease & 1 \\
\hline Acute bronchitis & ILD & 1 \\
\hline
\end{tabular}

COPD chronic obstructive pulmonary disease, ILD interstitial lung disease

Table 1 Demographic and clinical variables predictive of mortality in patients with haemoptysis

\begin{tabular}{|c|c|c|c|c|}
\hline & \multicolumn{2}{|c|}{ Univariate analysis } & \multicolumn{2}{|c|}{ Multivariate analysis } \\
\hline & OR $(95 \% \mathrm{CI})$ & $p$-value & OR $(95 \% \mathrm{Cl})$ & p-value \\
\hline \multicolumn{5}{|l|}{ Age (classes) } \\
\hline$<40$ years & Ref & Ref & & \\
\hline 40-54 years & $0.8(0.1-9.0)$ & 0.85 & & \\
\hline $55-70$ years & $3.3(0.4-26.4)$ & 0.25 & & \\
\hline$>70$ years & $9.5(1.3-70.5)$ & 0.03 & $5.0(0.8-32.7)$ & 0.10 \\
\hline Sex male & $2.3(1.3-4.1)$ & 0.005 & $1.3(0.2-7.4)$ & 0.76 \\
\hline \multicolumn{5}{|l|}{ Haemoptysis severity } \\
\hline Mild & Ref & Ref & & \\
\hline Moderate & $1.9(1.2-3.0)$ & 0.01 & & \\
\hline Severe & $0.6(0.1-4.6)$ & 0.61 & & \\
\hline \multicolumn{5}{|l|}{ Smoking history } \\
\hline$\geq 10$ pack/years & $3.5(1.8-6.7)$ & $<0.0001$ & $0.5(0.1-3.5)$ & 0.46 \\
\hline$\geq 30$ pack/years & $1.9(1.1-3.4)$ & 0.02 & & \\
\hline Number of recurrences & $0.2(0.1-0.7)$ & 0.009 & $0.6(0.2-2.3)$ & 0.46 \\
\hline \multicolumn{5}{|l|}{ Severity of recurrences } \\
\hline Mild & Ref & Ref & & \\
\hline Moderate & $3.6(1.2-10.8)$ & 0.02 & & \\
\hline Severe & $10.8(1.6-73.9)$ & 0.02 & & \\
\hline Antiplatelet therapy & $1.3(0.8-2.1)$ & 0.37 & & \\
\hline Anticoagulant therapy & $1.5(0.8-2.9)$ & 0.25 & & \\
\hline Antiplatelet + anticoagulant therapy & $1.3(0.8-2.1)$ & 0.24 & & \\
\hline Pneumonia/lung abscess & $0.7(0.3-1.3)$ & 0.20 & & \\
\hline Malignancy (primary and metastatic) & $15.6(9.2-26.5)$ & $<0.0001$ & $38.2(4.2-347.5)$ & 0.0001 \\
\hline Bronchiectasis & $0.1(0.0-0.5)$ & 0.004 & $0.3(0.0-3.7)$ & 0.37 \\
\hline Acute bronchitis & - & - & & \\
\hline Cryptogenic haemoptysis & $0.3(0.1-1.1)$ & 0.08 & & \\
\hline
\end{tabular}


diagnosed with idiopathic bleeding, in two patients with an exacerbation of chronic obstructive pulmonary disease (COPD) (both with a smoking history $>20$ pack/ years), and in four patients with pneumonia/lung abscess (two of them active smokers). In 7/22 (32\%) patients, the new diagnosis was determined during a recurrence: lung cancer was described in two patients with an initial diagnosis of pneumonia/lung abscess and in one with idiopathic bleeding. In two patients, an upper airways lesion was found after an initial diagnosis of acute bronchitis and idiopathic bleeding. An upper digestive haemorrhage and a pulmonary embolism were diagnosed in two patients initially diagnosed with cryptogenic haemoptysis and pneumonia, respectively.

Recurrent episodes of haemoptysis were recorded in 103/606 (17\%) patients (Table 3). The median (IQR) number of events was 1 (1-2). Among 103 patients with bronchiectasis, 28 (27.2\%) experienced recurrences (45 bleeding events). Recurrences were also recorded in $24 / 103(23.3 \%)$ with pulmonary malignancy $(22 / 103$ (21.4\%) with lung cancer (26 events) and 2/103 (1.9\%) with pulmonary metastasis (4 events), in 13/103 (12.6\%) with pneumonia/lung abscess (21 events). Bronchiectasis and pneumonia/lung abscess related recurrences were mostly mild (20 (71.4\%) and $13(100 \%)$ patients, respectively), whereas in case of lung cancer mild to moderate bleeding was found in 10 (45.5\%) patients. Two (7.1\%) severe recurrences were recorded in patients with both bronchiectasis and lung cancer.
The univariate analysis showed that bronchiectasis was significantly associated with the occurrence of a recurrence (OR: 2.6); p-value<0.0001) (Table 4). Anticoagulant, antiaggregant, and anticoagulant plus antiaggregant drugs were not significantly associated with an increased risk of mortality or recurrence (Table 5).

\section{Discussion}

To the best of our knowledge, this is the largest prospective study describing the long-term prognostic outcomes (18 months) of patients with haemoptysis.

An overall mortality rate of $13.7 \%$ was found; the number of deaths increased from 18.1 to $31 \%$ after one year of follow-up and then decreased to $8.4 \%$ at the end of the study period. Most of the deaths occurring during followup were related to the aetiology that caused the haemoptysis, with pulmonary neoplasms being the leading cause.

Malignancy, which was reported as the most frequent aetiology in patients with haemoptysis in several studies $[3-6,8,11]$, represents the only significant predictor of mortality in our study. Haemoptysis related to bronchiectasis, lower respiratory tract infections, and other less frequent aetiologies was not associated with an increase in mortality.

Two previous prospective studies with a small sample size and a follow-up period of 1.8-2.7 years described a slightly higher mortality rate $(19.5-22 \%)[7,8]$, mainly driven by malignancies [8]. More heterogeneous findings were reported by recently published European

Table 3 Haemoptysis recurrences and their severity in 103 patients related to the final diagnosis

\begin{tabular}{|c|c|c|c|c|c|}
\hline Final diagnosis & Events & Patients & Mild & Moderate & Severe \\
\hline Bronchiectasis, n (\%) & 45 & $28(27.2)$ & $20(71.4)$ & $6(21.4)$ & $2(7.1)$ \\
\hline Pulmonary malignancy, n (\%) & 30 & $24(23.3)$ & $11(36.6)$ & $11(36.6)$ & $2(0.8)$ \\
\hline Lung cancer, n (\%) & 26 & $22(21.4)$ & $10(45.5)$ & $10(45.5)$ & $2(9.1)$ \\
\hline Pulmonary metastasis, n (\%) & 4 & $2(1.9)$ & $1(50.0)$ & $1(50.0)$ & $0(0.0)$ \\
\hline Pneumonia/lung abscess, n (\%) & 21 & $13(12.6)$ & $13(100)$ & $0(0.0)$ & $0(0.0)$ \\
\hline COPD (exacerbation), n (\%) & 13 & $7(6.8)$ & $7(100.0)$ & $0(0.0)$ & $0(0.0)$ \\
\hline Acute bronchitis, n (\%) & 11 & $7(6.8)$ & $7(100.0)$ & $0(0.0)$ & $0(0.0)$ \\
\hline Cryptogenic haemoptysis, n (\%) & 10 & $6(5.8)$ & $5(83.3)$ & $1(16.7)$ & $0(0.0)$ \\
\hline Upper airways bleeding disease, n (\%) & 8 & $4(3.9)$ & $2(50.0)$ & $2(50.0)$ & $0(0.0)$ \\
\hline Post-tuberculosis sequelae, n (\%) & 8 & $3(2.9)$ & $2(66.7)$ & $1(33.3)$ & $0(0.0)$ \\
\hline Other pulmonary/bronchial vascular lesion, n (\%) & 5 & $2(1.9)$ & $1(50.0)$ & $0(0.0)$ & $1(50.0)$ \\
\hline Pulmonary embolism, n (\%) & 5 & $2(1.9)$ & $1(50.0)$ & $1(50.0)$ & $0(0.0)$ \\
\hline Atypical mycobacteriosis, n (\%) & 2 & $1(1.0)$ & $1(100.0)$ & $0(0.0)$ & $0(0.0)$ \\
\hline Active tuberculosis, n (\%) & 2 & $2(1.9)$ & $1(50.0)$ & $1(50.0)$ & $0(0.0)$ \\
\hline latrogenic or traumatic, n (\%) & 2 & $1(1.0)$ & $1(100.0)$ & $0(0.0)$ & $0(0.0)$ \\
\hline Tracheal granuloma, n (\%) & 2 & $1(1.0)$ & $1(100.0)$ & $0(0.0)$ & $0(0.0)$ \\
\hline Hematemesis, n (\%) & 1 & $1(1.0)$ & $0(0.0)$ & $1(100.0)$ & $0(0.0)$ \\
\hline Interstitial lung disease, n (\%) & 1 & $1(1.0)$ & $1(100.0)$ & $0(0.0)$ & $0(0.0)$ \\
\hline
\end{tabular}


Table 4 Demographic and clinical variables predictive of recurrence in patients with haemoptysis

\begin{tabular}{|c|c|c|c|c|}
\hline & \multicolumn{2}{|c|}{ Univariate analysis } & \multicolumn{2}{|c|}{ Multivariate analysis } \\
\hline & OR (95\% Cl) & $p$-value & OR $(95 \% \mathrm{Cl})$ & $p$-value \\
\hline \multicolumn{5}{|l|}{ Age (years) } \\
\hline$<40$ & Ref. & Ref. & & \\
\hline $40-54$ & $1.3(0.4-4.4)$ & 0.63 & & \\
\hline $55-70$ & $1.6(0.5-5.0)$ & 0.41 & & \\
\hline$>70$ & $1.9(0.7-5.7)$ & 0.23 & & \\
\hline Sex male & $0.9(0.6-1.3)$ & 0.51 & & \\
\hline \multicolumn{5}{|l|}{ Severity of haemoptysis } \\
\hline Mild & Ref. & Ref. & & \\
\hline Moderate & $1.2(0.8-2.0)$ & 0.39 & & \\
\hline Severe & $1.2(0.8-2.0)$ & 0.35 & & \\
\hline \multicolumn{5}{|l|}{ Smoking history } \\
\hline$\geq 10$ pack/years & $0.7(0.4-1.1)$ & 0.09 & & \\
\hline$\geq 30$ pack/years & $0.9(0.5-1.6)$ & 0.74 & & \\
\hline Antiplatelet therapy & $0.9(0.5-1.5)$ & 0.69 & & \\
\hline Anticoagulant therapy & $1.1(0.6-2.2)$ & 0.75 & & \\
\hline Antiplatelet + anticoagulant therapy & $1.0(0.6-1.6)$ & 0.98 & & \\
\hline Pneumonia/lung abscess & $0.7(0.4-1.3)$ & 0.25 & & \\
\hline Malignancy (primary and metastatic) & $1.1(0.6-1.9)$ & 0.79 & & \\
\hline Bronchiectasis & $2.6(1.5-4.3)$ & $<0.0001$ & & \\
\hline Acute bronchitis & $0.6(0.3-1.3)$ & 0.18 & & \\
\hline Cryptogenic haemoptysis & $1.0(0.5-2.1)$ & 0.94 & & \\
\hline
\end{tabular}

retrospective studies where lung cancer was the major cause of death, but the mortality rate ranged from 5.9 to $27 \%[2,6,10]$. Petersen et al. showed that older age, previous diagnosis of lung cancer, current/previous smoking history, and concomitant lung diseases are independent risk factors of death [10]. Notably, cryptogenic haemoptysis accounted for $80.5 \%$ of the diagnoses in this cohort, suggesting a suboptimal or difficult-to-perform diagnostic assessment [10].

The recurrence rate was $17 \%$, with bronchiectasis being the leading cause (27.2\%), and the most important predictor of recurrence. Bronchiectasis recurrences were mainly mild in contrast with those caused by lung malignancies (i.e., the second most frequent cause of recurrence), which were equally mild and moderate.

Few recent retrospective data on this topic are available in the scientific literature. In keeping with our findings, Fidan et al. and Ryuge et al. found bronchiectasis as the most frequent diagnosis in recurrent hemoptysis $[12,13]$. Abdulmalak et al. described recurrence rates of $16.6 \%$ and $16.1 \%$ during a three-year follow-up period in 2008 and 2009, respectively [2]. Cryptogenic haemoptysis, lower respiratory tract infection, and lung cancer were the most common aetiologies of recurrences, with cryptogenic haemoptysis representing $50 \%$ of all causes.
Choi et al., who described a recurrence rate of $19.1 \%$, demonstrated that aspergillosis, active bleeding, and blood clots during bronchoscopy during the first evaluation were significantly associated with the risk of recurrence; however, they analysed only patients with mild hemoptysis [14]. Similar findings were described by Lee et al., who found that active bleeding during bronchoscopy, smoking history $>40$ years, and hypertension were the main predictors of recurrence. Notably, the aetiology was not evaluated in this study [15].

As suggested by Lee et al., recurrences should alert physicians to undetected pathological findings $[16,17]$.

In seven patients of our cohort, a recurrence was associated with a new clinical and radiological assessment and to an aetiological change. Three patients initially diagnosed with idiopathic bleeding or pneumonia were subsequently diagnosed with lung cancer, in contrast to the study by Tsoumakidou et al., where lung cancer was not diagnosed in any patients with an initial aetiology other than lung cancer [7]. On the contrary, Abdulmalak et al. described the highest rate of lung cancer detection during the follow-up of patients with a diagnosis of respiratory infection (10.4\%) at the baseline evaluation [2].

In our study, four patients initially diagnosed with acute bronchitis and COPD exacerbation based on 
Table 5 Role played by antiplatelet and anticoagulant therapy, alone and combined, on recurrence and mortality in patients with haemoptysis

\begin{tabular}{|c|c|c|c|}
\hline Antiplatelet and anticoagulant therapy & No & Yes & p-value \\
\hline Recurrence, n (\%) & $68(20.1)$ & $35(18.8)$ & 0.72 \\
\hline \multicolumn{4}{|l|}{ Recurrence severity, n (\%) } \\
\hline Mild & $49(72.1)$ & $25(71.4)$ & \multirow[t]{3}{*}{0.46} \\
\hline Moderate & $17(25.0)$ & $7(20.0)$ & \\
\hline Severe & $2(2.9)$ & $3(8.6)$ & \\
\hline Median (IQR) n. recurrence events & $1(1-2)$ & $1(1-2)$ & 0.12 \\
\hline Deaths after 18 months of follow-up, n (\%) & $50(12.6)$ & $33(16.0)$ & 0.24 \\
\hline Deaths for the disease which caused haemoptysis, n (\%) & $31(62.0)$ & $21(63.6)$ & 1.00 \\
\hline \multicolumn{4}{|l|}{ Antiplatelet therapy } \\
\hline Recurrence, n (\%) & $80(20.2)$ & $23(18.0)$ & 0.58 \\
\hline \multicolumn{4}{|l|}{ Recurrence severity, $\mathrm{n}(\%)$} \\
\hline Mild & $59(73.8)$ & $15(65.2)$ & \multirow[t]{3}{*}{0.16} \\
\hline Moderate & $19(23.8)$ & $5(21.7)$ & \\
\hline Severe & $2(2.5)$ & $3(13.0)$ & \\
\hline Median (IQR) n. recurrence events & $1.5(1-2)$ & $1(1-2)$ & 0.04 \\
\hline Deaths after 18 months of follow-up, n (\%) & $60(13.0)$ & $23(16.0)$ & 0.37 \\
\hline Deaths for the disease which caused haemoptysis, n (\%) & $35(58.3)$ & $17(73.9)$ & 0.19 \\
\hline \multicolumn{4}{|l|}{ Anticoagulant therapy } \\
\hline Recurrence, n (\%) & $91(19.7)$ & $12(19.7)$ & 0.98 \\
\hline \multicolumn{4}{|l|}{ Recurrence severity, n (\%) } \\
\hline Mild & $64(70.3)$ & $10(83.3)$ & \multirow[t]{3}{*}{0.85} \\
\hline Moderate & $22(24.2)$ & $2(16.7)$ & \\
\hline Severe & $5(5.5)$ & $0(0.0)$ & \\
\hline Median (IQR) n. recurrence events & $1(1-2)$ & $2(1-2)$ & 0.66 \\
\hline Deaths after 18 months of follow-up, n (\%) & $71(13.2)$ & $12(18.5)$ & 0.24 \\
\hline Deaths for the disease which caused haemoptysis, n (\%) & $47(66.2)$ & $5(41.7)$ & 0.10 \\
\hline
\end{tabular}

clinical and chest X-ray findings were subsequently diagnosed with bronchiectasis.

The present scientific evidence suggests the importance of a clinical and radiological follow-up in patients with bleeding of unknown origin and related to an acute lower respiratory tract infection, as well as a more accurate radiological assessment (i.e., chest CT) in patients with haemoptysis and risk factors for bronchiectasis [18].

Very little data could be retrieved on the impact of antiplatelet or anticoagulant therapies on long-term prognostic outcomes. In a large retrospective cohort study, Lee et al. failed to demonstrate a potential role of aspirin on recurrence [15]. Similar findings were shown by Ryuge et al., who studied the mechanism of haemoptysis relapse in patients who underwent BAE; they demonstrated that antiplatelet and anticoagulant therapy did not increase recanalisation, i.e. the most frequent mechanism underlying re-hemoptysis [13]. We have prospectively demonstrated that these drugs, individually or in combination, did not change the risk of death and recurrence.
Some study limitations can be found. The observational nature cannot help discriminate the role played by some medical conditions for the background noise of confounders. However, for ethical reasons, it is currently the best methodological approach. Differences in some standard operating procedures in the recruited centres could affect some results in terms of diagnostic accuracy. However, the recruited hospitals were national reference centres, and thus, operational variability should be not relevant. Subgroup analyses by aetiology could have affected the statistical power of some findings; future studies focused on specific aetiologies could confirm our novel results.

\section{Conclusions}

In conclusion, our study shows a low mortality rate in patients with haemoptysis followed-up for a long period. Pulmonary malignancy is the main aetiology and the main predictor of death in these patients, whereas bronchiectasis is the most frequent diagnosis associated with recurrence. Antiplatelet and/or anticoagulant therapy do 
not change the risk of death or recurrence. Follow-up is recommended in patients initially diagnosed with lower airways infections and those with idiopathic bleeding, in order to detect new or misdiagnosed lung malignancies.

\author{
Abbreviations \\ ml: Millilitres; IQR: Interquartile range; COPD: Chronic obstructive pulmonary \\ disease.
}

\section{Acknowledgements}

Not applicable.

\section{Authors' contributions}

Guarantor: MM is the guarantor of the content of the manuscript, including the data and analysis. Author contribution: MM: contributions to the conception and design of the work, acquisition, and interpretation of data for the work; drafting the work; final approval of the version to be published; agreement to be accountable for all aspects of the work in ensuring that questions related to the accuracy or integrity of any part of the work are appropriately investigated and resolved. PC: contributions to the conception and design of the work, acquisition, and interpretation of data for the work; revising the manuscript critically for important intellectual content; final approval of the version to be published; agreement to be accountable for all aspects of the work in ensuring that questions related to the accuracy or integrity of any part of the work are appropriately investigated and resolved. LS: contributions to the analysis and interpretation of data for the work; revising the manuscript critically for important intellectual content; final approval of the version to be published; agreement to be accountable for all aspects of the work in ensuring that questions related to the accuracy or integrity of any part of the work are appropriately investigated and resolved. GS: contributions to the conception or design of the work, analysis and interpretation of data for the work; drafting the work; final approval of the version to be published; agreement to be accountable for all aspects of the work in ensuring that questions related to the accuracy or integrity of any part of the work are appropriately investigated and resolved-GC, MP, FT, PP, AF, AA, SC, SG, MB, ACC, SM, FA, SDP, SC: acquisition of data for the work; revising the manuscript critically for important intellectual content; final approval of the version to be published; agreement to be accountable for all aspects of the work in ensuring that questions related to the accuracy or integrity of any part of the work are appropriately investigated and resolved. All authors read and approved the final manuscript.

\section{Funding}

None.

\section{Availability of data and materials}

The datasets used and/or analysed during the current study are available from the corresponding author on reasonable request.

\section{Declarations}

Ethics approval and consent to participate

Resolution n. 665 (18/07/2013), Comitato Etico Ospedale San Paolo, Milan, taly.

\section{Consent for publication}

Not applicable.

\section{Competing interests}

The authors declare that they have no competing interests.

\section{Author details}

${ }^{1}$ Respiratory Unit, ASST Santi Paolo e Carlo, San Paolo Hospital, Department of Health Sciences, Università degli Studi di Milano, Via A. Di Rudinì n.8, 20142 Milan, Italy. ${ }^{2}$ UOC Pneumologia, ASST Lodi, Lodi, Italy. ${ }^{3}$ UO Pneumologia ed Endoscopia Toracica, Azienda Ospedaliero Universitaria di Parma, Parma, Italy. ${ }^{4}$ UOS Servizio Pneumologia, Ospedale di Codogno, ASST Lodi, Codogno
(Lodi), Italy. ${ }^{5}$ Lung Disease Unit, Dept of Clinical and Experimental Medicine, University of Sassari, Sassari, Italy. ${ }^{6}$ Pulmonary Disease Unit, Department of Internal Medicine, Azienda Ospedali Riuniti, Department of Biomedical Sciences and Public Health, Università Politecnica Delle Marche, Ancona, Italy. ${ }^{7}$ UO Medicina Interna, AUSL Modena, Ospedale di Carpi, Carpi (Modena), Italy. ${ }^{8}$ UO Pneumologia, Ospedale P. Pederzoli, Peschiera del Garda (Verona), Italy. ${ }^{9}$ Clinical Epidemiology and Medical Statistics Unit, Dept of Medical, Surgical and Experimental Sciences, University of Sassari, Sassari, Italy.

Received: 14 April 2021 Accepted: 23 July 2021

Published online: 04 August 2021

\section{References}

1. Mondoni M, Sferrazza Papa GF, Sotgiu G, Carlucci P, Pellegrino GM, Centanni S. Haemoptysis: a frequent diagnostic challenge. Eur Respir J. 2016:47(1):348-50.

2. Abdulmalak C, Cottenet J, Beltramo G, et al. Haemoptysis in adults: a 5-year study using the French nationwide hospital administrative database. Eur Respir J. 2015;46(2):503-11.

3. Quigley N, Gagnon S, Fortin M. Aetiology, diagnosis and treatment of moderate-to-severe haemoptysis in a North American academic centre. ERJ Open Res. 2020;6(4):00204-2020

4. Vanni S, Bianchi S, Bigiarini S, et al. Management of patients presenting with haemoptysis to a Tertiary Care Italian Emergency Department: the Florence Haemoptysis Score (FLHASc). Intern Emerg Med. 2018;13(3):397-404.

5. Mondoni M, Carlucci $P$, Job S, et al. Observational, multicentre study on the epidemiology of haemoptysis. Eur Respir J. 2018;51(1):1701813.

6. Soares Pires F, Teixeira N, Coelho F, Damas C. Hemoptysis-etiology, evaluation and treatment in a university hospital. Rev Port Pneumol. 2011;17(1):7-14.

7. Tsoumakidou M, Chrysofakis G, Tsiligianni I, Maltezakis G, Siafakas NM, Tzanakis N. A prospective analysis of 184 hemoptysis cases: diagnostic impact of chest X-ray, computed tomography, bronchoscopy. Respiration. 2006;73(6):808-14.

8. Uzun $O$, Atasoy Y, Findik S, Atici AG, Erkan L. A prospective evaluation of hemoptysis cases in a tertiary referral hospital. Clin Respir J. 2010;4(3):131-8.

9. Arooj P, Bredin E, Henry MT, et al. Bronchoscopy in the investigation of outpatients with hemoptysis at a lung cancer clinic. Respir Med. 2018;139:1-5

10. Petersen CL, Weinreich UM. Five-year follow-up of hemoptysis with no malignancy suspected on chest computed tomography: recurrence, lung cancer and mortality. Eur Clin Respir J. 2019;6(1):1616519.

11. Hirshberg B, Biran I, Glazer M, Kramer MR. Hemoptysis: etiology, evaluation, and outcome in a tertiary referral hospital. Chest. 1997;112(2):440-4.

12. Fidan A, Ozdoğan S, Oruç O, Salepçi B, Ocal Z, Cağlayan B. Hemoptysis: a retrospective analysis of 108 cases. Respir Med. 2002;96(9):677-80.

13. Ryuge $M$, Hara M, Hiroe T, et al. Mechanisms of recurrent haemoptysis after super-selective bronchial artery coil embolisation: a single-centre retrospective observational study. Eur Radiol. 2018:29(2):70.

14. Choi J, Baik JH, Kim CH, et al. Long-term outcomes and prognostic factors in patients with mild hemoptysis. Am J Emerg Med. 2018;36(7):1160-5.

15. Lee MK, Kim SH, Yong SJ, et al. Moderate hemoptysis: recurrent hemoptysis and mortality according to bronchial artery embolization. Clin Respir J. 2015;9(1):53-64

16. Lee YJ, Lee SM, Park JS, et al. The clinical implications of bronchoscopy in hemoptysis patients with no explainable lesions in computed tomography. Respir Med. 2012;106(3):413-9.

17. Mondoni M, Carlucci P, Cipolla G, et al. Bronchoscopy to assess patients with hemoptysis: which is the optimal timing? BMC Pulm Med. 2019;19(1):36.

18. Expert Panel on Thoracic Imaging, Olsen KM, Manouchehr-Pour S, Donnelly EF, et al. ACR appropriateness criteria hemoptysis. J Am Coll Radiol. 2020;17(5S):S148-S159.

\section{Publisher's Note}

Springer Nature remains neutral with regard to jurisdictional claims in published maps and institutional affiliations. 